\title{
Neoadjuvant Chemotherapy or Primary Surgery in Stage IIIC or IV Ovarian Cancer
}

\author{
Ignace Vergote, M.D., Ph.D., Claes G. Tropé, M.D., Ph.D., \\ Frédéric Amant, M.D., Ph.D., Gunnar B. Kristensen, M.D., Ph.D., \\ Tom Ehlen, M.D., Nick Johnson, M.D., René H.M. Verheijen, M.D., Ph.D., \\ Maria E.L. van der Burg, M.D., Ph.D., Angel J. Lacave, M.D., \\ Pierluigi Benedetti Panici, M.D., Ph.D., Gemma G. Kenter, M.D., Ph.D., \\ Antonio Casado, M.D., Cesar Mendiola, M.D., Ph.D., Corneel Coens, M.Sc., \\ Leen Verleye, M.D., Gavin C.E. Stuart, M.D., Sergio Pecorelli, M.D., Ph.D., \\ and Nick S. Reed, M.D., for the European Organization for Research and \\ Treatment of Cancer-Gynaecological Cancer Group and the NCIC Clinical Trials \\ Group* - a Gynecologic Cancer Intergroup Collaboration
}

ABSTRACT

\section{BACKGROUND}

Primary debulking surgery before initiation of chemotherapy has been the standard of care for patients with advanced ovarian cancer.

\section{METHODS}

We randomly assigned patients with stage IIIC or IV epithelial ovarian carcinoma, fallopian-tube carcinoma, or primary peritoneal carcinoma to primary debulking surgery followed by platinum-based chemotherapy or to neoadjuvant platinum-based chemotherapy followed by debulking surgery (so-called interval debulking surgery).

\section{RESULTS}

Of the 670 patients randomly assigned to a study treatment, 632 (94.3\%) were eligible and started the treatment. The majority of these patients had extensive stage IIIC or IV disease at primary debulking surgery (metastatic lesions that were larger than $5 \mathrm{~cm}$ in diameter in $74.5 \%$ of patients and larger than $10 \mathrm{~cm}$ in $61.6 \%)$. The largest residual tumor was $1 \mathrm{~cm}$ or less in diameter in $41.6 \%$ of patients after primary debulking and in $80.6 \%$ of patients after interval debulking. Postoperative rates of adverse effects and mortality tended to be higher after primary debulking than after interval debulking. The hazard ratio for death (intention-to-treat analysis) in the group assigned to neoadjuvant chemotherapy followed by interval debulking, as compared with the group assigned to primary debulking surgery followed by chemotherapy, was 0.98 ( $90 \%$ confidence interval [CI], 0.84 to 1.13 ; $\mathrm{P}=0.01$ for noninferiority), and the hazard ratio for progressive disease was 1.01 (90\% CI, 0.89 to 1.15). Complete resection of all macroscopic disease (at primary or interval surgery) was the strongest independent variable in predicting overall survival.

\section{CONCLUSIONS}

Neoadjuvant chemotherapy followed by interval debulking surgery was not inferior to primary debulking surgery followed by chemotherapy as a treatment option for patients with bulky stage IIIC or IV ovarian carcinoma in this study. Complete resection of all macroscopic disease, whether performed as primary treatment or after neoadjuvant chemotherapy, remains the objective whenever cytoreductive surgery is performed. (Funded by the National Cancer Institute; ClinicalTrials.gov number, NCT00003636.)
From the University Hospitals Leuven, Leuven (I.V., F.A.), and the European Organization for Research and Treatment of Cancer Headquarters, Brussels (C.C., L.V.) - both in Belgium; Norwegian Radium Hospital and the Institute of Medical Informatics, Oslo (C.G.T., G.B.K.); University of British Columbia, Vancouver, Canada (T.E., G.C.E.S.); Royal United Hospital, Bath (N.J.), and Gartnavel General Hospital and Beatson Oncology Center, Glasgow (N.S.R.) - both in the United Kingdom; Vrije Universiteit Medical Center, Amsterdam (R.H.M.V.), Erasmus MC University Medical Center Rotterdam, Rotterdam (M.E.L.B.), and Leiden University Medical Center, Leiden (G.G.K.) - all in the Netherlands; Hospital Universitario Central de Asturias, Oviedo, Spain (A.J.L.); University of Rome La Sapienza, Rome (P.B.P.), and the University of Brescia, Brescia (S.P.) - both in Italy; and Hospital Universitario San Carlos (A.C.) and Hospital Universitario 12 de Octubre (C.M.) both in Madrid. Address reprint requests to Dr. Vergote at University Hospitals, K.U. Leuven Division of Gynecologic Oncology, Department of Obstetrics and Gynecology, Herestraat 49, B-3000 Leuven, Belgium, or at ignace.vergote@uzleuven.be.

*Other collaborators are listed in the Appendix.

N Engl J Med 2010;363:943-53.

Copyright @ 2010 Massachusetts Medical Society. 
I N MOST WOMEN WITH OVARIAN CARCINO$\mathrm{ma}$, the disease is not diagnosed until it is at an advanced stage. Primary cytoreductive surgery is considered the standard of care for advanced ovarian carcinoma. ${ }^{1-4}$ However, data from prospective, randomized, controlled trials assessing the role of primary surgery in the treatment of such cases are lacking. Interval debulking surgery has not been viewed as beneficial in women with residual tumor that exceeds $1 \mathrm{~cm}$ in diameter after primary debulking surgery performed with the objective of maximal surgical effort by a gynecologic oncologist. ${ }^{5-7}$ As an alternative to primary debulking surgery followed by chemotherapy, some authors have investigated the use of neoadjuvant chemotherapy before cytoreductive surgery. However, results of a meta-analysis involving 835 patients suggested that neoadjuvant chemotherapy, as compared with primary debulking surgery, was associated with a worse outcome. ${ }^{8}$

We report on a randomized trial in which we compared primary debulking surgery followed by platinum-based chemotherapy and platinum-based neoadjuvant chemotherapy followed by interval debulking surgery and additional platinum-based chemotherapy in women with advanced ovarian carcinoma.

\section{METHODS}

\section{PATIENTS}

Eligible patients had biopsy-proven stage IIIC or IV invasive epithelial ovarian carcinoma, primary peritoneal carcinoma, or fallopian-tube carcinoma. If a biopsy specimen was not available, a fine-needle aspirate showing an adenocarcinoma was acceptable under the following conditions: the presence of a pelvic (ovarian) mass; the presence of metastases outside the pelvis measuring at least $2 \mathrm{~cm}$ in diameter (as noted during diagnostic laparoscopy or laparotomy or on computed tomography $[\mathrm{CT}])$; regional lymph-node metastasis or proof of stage IV disease; and a ratio of cancer antigen 125 (CA-125, measured in kilounits per liter) to carcinoembryonic antigen (CEA, measured in nanograms per milliliter) that was greater than 25. The CA-125:CEA ratio has been shown to be useful for ruling out primary gastrointestinal tumors that have metastasized to the peritoneum, the ovaries, or both. ${ }^{9}$ If the serum CA-125: CEA ratio was 25 or lower, results of a barium enema (or colonoscopy), gastroscopy (or radio- logic examination of the stomach), and mammography (performed within 6 weeks before randomization) had to be negative for the presence of a primary tumor. Additional prerandomization requirements included a World Health Organization (WHO) performance status of 0 (asymptomatic) to 2 (symptomatic, in bed for less than half the day $)^{10}$ and the absence of serious disabling diseases that would contraindicate primary cytoreductive surgery or platinum-based chemotherapy. (Other inclusion criteria are listed in the Supplementary Appendix, available with the full text of this article at NEJM.org.) Before receiving treatment, all patients provided written informed consent. Because of an allegation of ethical irregularities at one of the centers with regard to another European Organization for Research and Treatment of Cancer (EORTC) protocol, all the patients from that center who were enrolled in this study were excluded from the analysis.

\section{STUDY DESIGN}

Patients had to start the assigned treatment within 3 weeks after the initial biopsy or fine-needle aspiration. The biopsy could be image-guided or carried out during laparoscopy or laparotomy. Patients who underwent laparotomy or laparoscopy were not allowed to undergo any procedures other than the diagnostic biopsies. Randomization was done centrally at the EORTC headquarters after stratification, with the use of a minimization technique to stratify for institution, method of biopsy (image-guided, laparoscopy, laparotomy, or fine-needle aspiration), tumor stage (IIIC or IV), and largest preoperative tumor size (excluding ovaries) $(\leq 5 \mathrm{~cm},>5$ to $10 \mathrm{~cm},>10$ to $20 \mathrm{~cm}$, or $>20 \mathrm{~cm})$.

Patients were randomly assigned either to primary debulking surgery followed by at least six courses of platinum-based chemotherapy or to three courses of neoadjuvant platinum-based chemotherapy followed by interval debulking surgery in all patients with a response or stable disease, followed in turn by at least three courses of platinum-based chemotherapy. In patients randomly assigned to primary debulking whose surgery was completed without optimal cytoreduction, interval debulking surgery was permitted if stable disease or a response was documented, and these patients were included in the primary-surgery group for analyses. After the results of the Gynecologic Oncology Group trial (GOG-152) 
(NCT00002568) were published, ${ }^{6}$ interval debulking surgery was no longer recommended for patients in whom optimal cytoreduction was not achieved despite a maximal effort at primary debulking surgery. Data on the timing of interval debulking surgery and chemotherapy, chemotherapy regimens, and assessments are provided in the Supplementary Appendix. All surgical procedures had to be performed by qualified gynecologic oncologists who were appointed by the individual institutions before the start of the study, and all patients were evaluated for eligibility before randomization, with no additional selection criteria (including resectability) imposed by the surgeon. No CT or laparoscopic scoring systems were used in the selection of the patients.

The study was designed and the manuscript written by the first author in cooperation with the other authors. Data were gathered at the EORTC headquarters and analyzed in cooperation with the authors by the EORTC statistician, who vouches for the accuracy of the data and the analyses. The decision to submit the manuscript for publication was made by the authors in agreement with the EORTC-Gynaecological Cancer Group (EORTC-GCG) and the National Cancer Institute of Canada (NCIC) Clinical Trials Group. The study was approved by the EORTC Protocol Review Committee, the NCIC Clinical Trials Group Clinical Trials Committee, and the institutional review board of each participating institution. An independent data and safety monitoring committee was appointed to monitor the recruitment rate, the potential toxicity of the treatments, and the optimal percentage of debulking. The drugs administered for adjuvant chemotherapy were purchased by the individual institutions. The study was conducted in accordance with the protocol as amended. (The trial protocol is available at NEJM.org.)

\section{EVALUATION AND FOLLOW-UP}

Patients filled out two EORTC quality-of-life questionnaires at five time points during the study: EORTC QLQ-C30 (http://groups.eortc.be/qol/ questionnaires_qlqc30.htm) and QLQ-Ov28 (http:// groups.eortc.be/qol/downloads/modules/specimen _20qlq_ov28.pdf).

Tumor response during chemotherapy was evaluated according to the WHO criteria. ${ }^{11}$ In addition, progression of disease after first-line chemotherapy was defined by an increase by a factor of at least 2 in the nadir serum CA-125 level according to the Gynaecologic Cancer Intergroup criteria. ${ }^{12}$

\section{STATISTICAL ANALYSIS}

The primary end point of the study was overall survival. The group undergoing primary debulking surgery was considered to be the standardtreatment group. On the basis of the earlier experience of the EORTC institutions, about $50 \%$ of patients with stage IIIC or IV ovarian carcinoma who underwent debulking surgery had a residual tumor size of $1 \mathrm{~cm}$ or less and had a median survival of 36 months. ${ }^{13}$ On the basis of a previous EORTC trial of interval debulking surgery, median survival among the patients with suboptimal primary debulking who underwent interval surgery was expected to be 26 months. ${ }^{5}$ Thus, the median survival of the whole group of patients randomly assigned to primary surgery was expected to be 31 months. With an accrual time of 4 years and a minimum follow-up period of 3 years, 498 events (704 patients) were required to show noninferiority of interval debulking surgery as compared with primary surgery, with a one-sided type I error rate of 0.05 and a power of $80 \%$. A hazard ratio of less than 1.25 was considered to indicate noninferiority. Secondary end points were adverse effects, quality of life, and progression-free survival. No interim analyses were planned or conducted.

The analysis was planned to be performed according to the intention-to-treat principle: all randomly assigned patients were included in the primary analysis, regardless of whether they were eligible and whether they could be evaluated. A secondary analysis was based on the treatment actually received. For definitions of overall and progression-free survival, see the Supplementary Appendix. Overall and progression-free survival rates were estimated by means of the KaplanMeier method, and overall survival rates in the two groups were compared by means of the logrank test, with a noninferiority ratio of 0.8 . Multivariate time-to-event analysis was performed with the use of a Cox proportional-hazards model and univariate screening followed by a stepwise variable-selection procedure. ${ }^{14}$ Adverse events were reported in contingency tables with the use of the National Cancer Institute Common Toxicity Criteria, version 2.0 (http:// ctep.cancer.gov/protocoldevelopment/electronic_ 


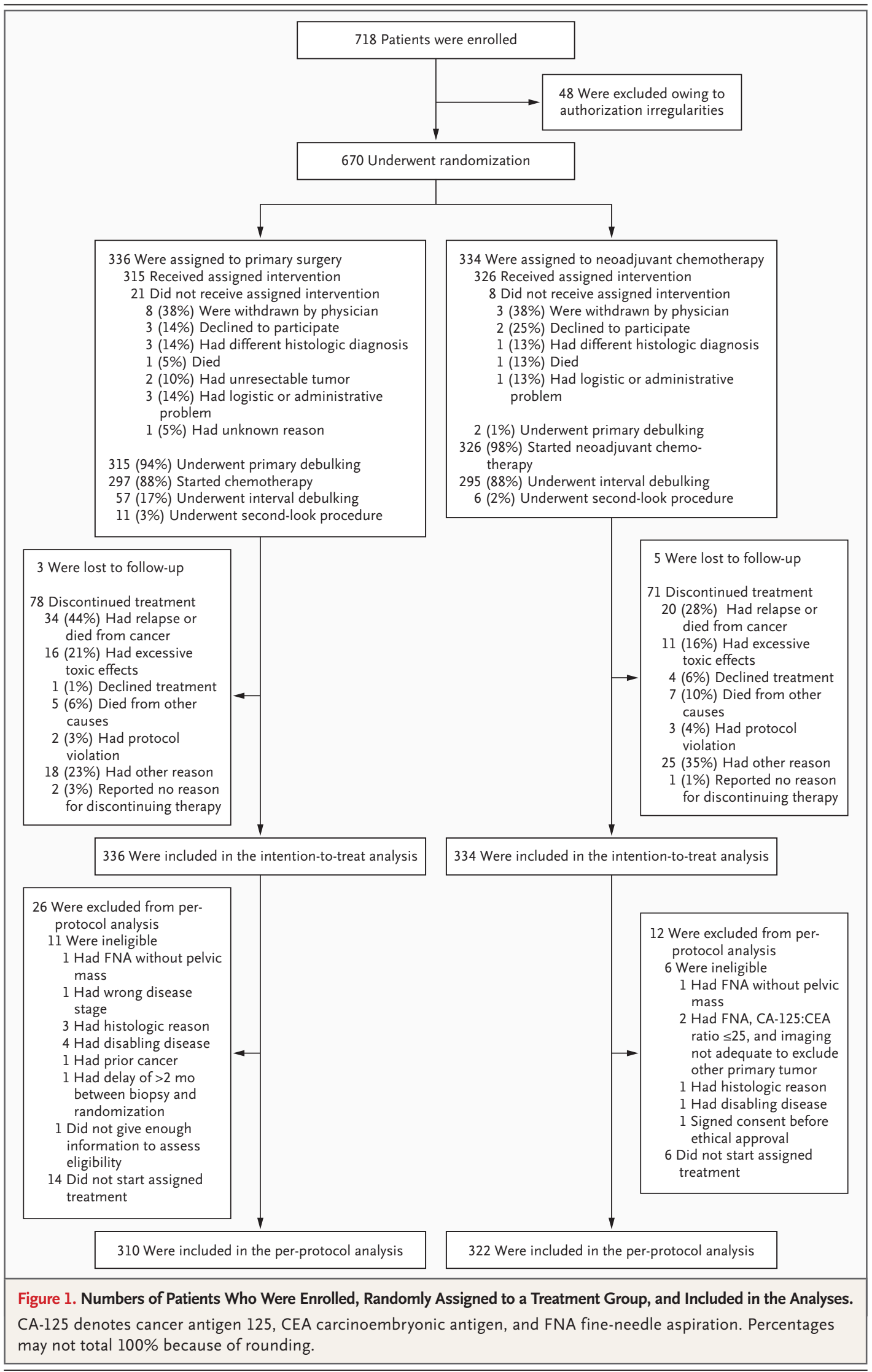


applications/docs/ctcv20_4-30-992.pdf); comparisons between treatment groups were made with the use of the log-rank test for trend. Adverse effects were regarded as postoperative if they occurred within 28 days after surgery. The largest metastases before randomization were measured during diagnostic laparoscopy or laparotomy, and if neither of these tests was done, their size was determined on the basis of CT findings.

\section{RESULTS}

CHARACTERISTICS OF THE PATIENTS

AND TREATMENT RECEIVED

From September 1998 through December 2006, a total of 718 patients were enrolled in the study; 48 patients were excluded because of potential authorization irregularities at one institution (Fig. 1). The remaining 670 patients were randomly assigned to treatment at 59 institutions (median accrual per institution, 5 patients; range, 1 to 125 ) (Fig. 1).

The results of the study were similar whether the 48 patients from the one center with possible irregularities were included or excluded. The requisite number of events was reached in August 2008 (median follow-up, 4.7 years).

The baseline characteristics of the patients were well balanced between the two treatment groups (Table 1). Details regarding residual tumor size, size of largest residual tumor per country, type of surgery, type of chemotherapy and number of courses, and time to initiation (or reinitiation) of chemotherapy are summarized in Table 1 in the Supplementary Appendix. The residual tumor size was $1 \mathrm{~cm}$ or smaller after primary debulking surgery in $41.6 \%$ of patients and after interval debulking surgery in $80.6 \%$ of patients.

After debulking surgery, the primary diagnosis changed in 11 patients (3.3\%) assigned to primary debulking surgery and in 7 patients (2.1\%) assigned to neoadjuvant chemotherapy followed by interval debulking surgery ( 5 carcinosarcomas, 4 endometrial carcinomas, 2 gastrointestinal tumors, 2 borderline tumors of the ovary, 1 cervical adenocarcinoma, 1 stage IC ovarian carcinoma, 1 teratoma, 1 rhabdomyosarcoma, and 1 pseudomyxoma).

The diaphragm, abdominal peritoneum, and pelvis (pouch of Douglas, uterus, bladder, rectum, and sigmoid) were the most frequent sites of residual tumor after both primary debulking and interval debulking surgery. Details on the size and site of the metastases before and after primary and interval debulking surgery, as well as the debulking rates after interval debulking in patients in the primary-surgery group, are summarized in the Supplementary Appendix. Within each country, there was a strong correlation between the rates of optimal debulking at primary debulking surgery and at interval debulking surgery $(r=0.92)$.

\section{PERIOPERATIVE AND POSTOPERATIVE MORBIDITY, MORTALITY, AND QUALITY OF LIFE}

Perioperative and postoperative morbidity and mortality are summarized in Table 1 in the Supplementary Appendix. Postoperative death (defined as death $<28$ days after surgery) occurred in $2.5 \%$ of patients in the primary-surgery group and in $0.7 \%$ of patients in the neoadjuvant-chemotherapy group. Grade 3 or 4 hemorrhage occurred in $7.4 \%$ of patients after primary debulking and in $4.1 \%$ after interval debulking, infection in $8.1 \%$ and $1.7 \%$, respectively, and venous complications in $2.6 \%$ and $0 \%$, respectively. Analyses comparing the perioperative and postoperative characteristics of the two groups were not performed because the groups were unequal - that is, not all patients who were randomly assigned to primary debulking underwent primary debulking surgery, and not all patients assigned to neoadjuvant chemotherapy underwent interval debulking surgery.

At none of the assessment times were the differences in the QLQ-C30 global health scores significant. The overall test for a treatment effect on global health was also not significant.

\section{OVERALL SURVIVAL AND PROGRESSION-FREE SURVIVAL}

Overall survival was similar in the two groups in the intention-to-treat analyses (Fig. 2A), as was progression-free survival (Fig. 1 in the Supplementary Appendix). The median overall survival was 29 months in the primary-surgery group and 30 months in the neoadjuvant-chemotherapy group, and the median progression-free survival in both groups was 12 months. On the basis of the intention-to-treat analysis, the hazard ratio for death in the group assigned to neoadjuvant chemotherapy followed by interval debulking, as compared with the group assigned to primary debulking, was $0.98(90 \%$ confidence interval [CI], 0.84 to 1.13; $\mathrm{P}=0.01$ for noninferiority), and the hazard 
ratio for progressive disease was 1.01 (90\% CI, 0.89 to 1.15 ). The analysis according to treatment actually received (per-protocol analysis) showed similar results for overall survival (hazard ratio for death, $1.00 ; 90 \%$ CI, 0.85 to $1.16 ; \mathrm{P}=0.01$ for noninferiority) (Fig. 2 in the Supplementary Appendix). Figure $2 \mathrm{~B}$ shows overall survival according to treatment group and amount of residual tumor (per-protocol analysis). Overall survival in the group of patients who underwent primary debulking surgery initially and then interval debulking surgery was similar to that in the group of patients who were randomly assigned to neoadjuvant chemotherapy (Fig. 3 in the Supplementary Appendix).

In a post hoc attempt to identify subgroups

Table 1. Baseline Characteristics of the Patients.

Characteristic

Age - yr

Median

Range

WHO performance status - no. (\%)*

0

1

Missing data

Histologic type - no. (\%)

Serous

Mucinous

Clear-cell

Endometrioid

Undifferentiated

Mixed

Other or unknown

Histologic grade - no. (\%)

Well differentiated

Moderately differentiated

Poorly differentiated

Unknown

Stage - no. (\%)

IIIC

IV

Other

Malignant pleural effusion - no. (\%)

No

Yes

Method of biopsy - no. (\%)

Laparotomy

Laparoscopy

Image guidance

Fine-needle aspiration

Missing data
Primary Debulking Surgery ( $N=336)$

62

25-86

$153(45.5)$

$141(42.0)$

40 (11.9)

$2(0.6)$

$220(65.5)$

$194(58.1)$

$8(2.4)$

$11(3.3)$

$6(1.8)$

4 (1.2)

$11(3.3)$

$5(1.5)$

$69(20.5)$

90 (26.9)

$3(0.9)$

0

$19(5.7)$

$30(9.0)$

14 (4.2)

$10(3.0)$

57 (17.0)

$41(12.3)$

145 (43.2)

130 (38.9)

$120(35.7)$

$153(45.8)$

257 (76.5)

$253(75.7)$

77 (22.9)

$81(24.3)$

$2(0.6)$

$285(84.8)$

$272(81.4)$

$51(15.2)$

$62(18.6)$

$12(3.6)$

$12(3.6)$

$104(31.0)$

116 (34.7)

$76(22.6)$

$53(15.9)$

$142(42.3)$

$153(45.8)$

$2(0.6)$

0 


\begin{tabular}{|c|c|c|}
\hline Characteristic & $\begin{array}{l}\text { Primary Debulking Surgery } \\
\qquad(\mathrm{N}=336)\end{array}$ & $\begin{array}{l}\text { Neoadjuvant Chemotherapy } \\
\qquad(\mathrm{N}=334)\end{array}$ \\
\hline \multicolumn{3}{|l|}{ Primary tumor — no. (\%) } \\
\hline Epithelial ovarian & $293(87.2)$ & $283(84.7)$ \\
\hline Peritoneal & $22(6.5)$ & $26(7.8)$ \\
\hline Fallopian tube & 0 & $4(1.2)$ \\
\hline Adenocarcinoma & $17(5.1)$ & $20(6.0)$ \\
\hline Missing data & $4(1.2)$ & $1(0.3)$ \\
\hline \multicolumn{3}{|l|}{ Serum CA-125 at entry $(\mathrm{U} / \mathrm{ml})$} \\
\hline Median & 1130 & 1180 \\
\hline Range & $16.0-27,185$ & $15.0-41,456$ \\
\hline Serum CA-125 >30 U/ml - no. (\%) & $330(98.2)$ & $330(98.8)$ \\
\hline \multicolumn{3}{|c|}{ Largest metastatic tumor at randomization — no. (\%) } \\
\hline $0 \mathrm{~cm}$ & $2(0.6)$ & $1(0.3)$ \\
\hline$>0-2 \mathrm{~cm}$ & $2(0.6)$ & $9(2.7)$ \\
\hline$>2-5 \mathrm{~cm}$ & $90(26.8)$ & $85(25.4)$ \\
\hline$>5-10 \mathrm{~cm}$ & $90(26.8)$ & $88(26.3)$ \\
\hline$>10-20 \mathrm{~cm}$ & $105(31.3)$ & $113(33.8)$ \\
\hline$>20 \mathrm{~cm}$ & $26(7.7)$ & $24(7.2)$ \\
\hline Missing data & $21(6.3)$ & $14(4.2)$ \\
\hline \multicolumn{3}{|c|}{ Size of metastases at the time of surgery - no. (\%) $\dagger$} \\
\hline No metastasis & $1 / 310(0.3)$ & $14 / 322(4.3)$ \\
\hline$>0-1 \mathrm{~cm}$ & $2 / 310(0.6)$ & $36 / 322(11.2)$ \\
\hline$>1-2 \mathrm{~cm}$ & $14 / 310(4.5)$ & $40 / 322(12.4)$ \\
\hline$>2-5 \mathrm{~cm}$ & $50 / 310(16.1)$ & $74 / 322(23.0)$ \\
\hline$>5-10 \mathrm{~cm}$ & $40 / 310(12.9)$ & $42 / 322(13.0)$ \\
\hline$>10 \mathrm{~cm}$ & $191 / 310(61.6)$ & $78 / 322(24.2)$ \\
\hline Missing data & $12 / 310(3.9)$ & $38 / 322(11.8)$ \\
\hline
\end{tabular}

* WHO denotes World Health Organization.

$\uparrow$ The per-protocol population included only eligible patients who actually underwent primary debulking surgery in the primary-surgery group (310 patients) and patients who actually started chemotherapy in the neoadjuvant-chemotherapy group (322).

of patients in which one of the study treatments tended to be associated with better overall survival, we analyzed the hazard plots in relation to age, the International Federation of Gynecology and Obstetrics (FIGO) stage, WHO performance status, histologic type, and presence or absence of pleural fluid (Fig. 4 to 8 in the Supplementary Appendix). In none of the subgroups was there apparent superiority of one of the treatments. When we evaluated the outcome of debulking to $1 \mathrm{~cm}$ or less according to country, no significant differences were noted between the treatment groups (Fig. 9 in the Supplementary Appendix). When the patients who were randomly assigned by the EORTC-GCG (586 patients) were compared with those randomly assigned by the NCIC Clinical Trials Group (84 patients), the median overall survival was similar (28 and 34 months, respectively). This result is noteworthy, since the proportions of patients with no residual tumor after primary debulking surgery and after interval debulking surgery tended to be higher in the subgroup randomly assigned by the EORTC-GCG ( $20.4 \%$ and $50.0 \%$, respectively) than in the sub- 


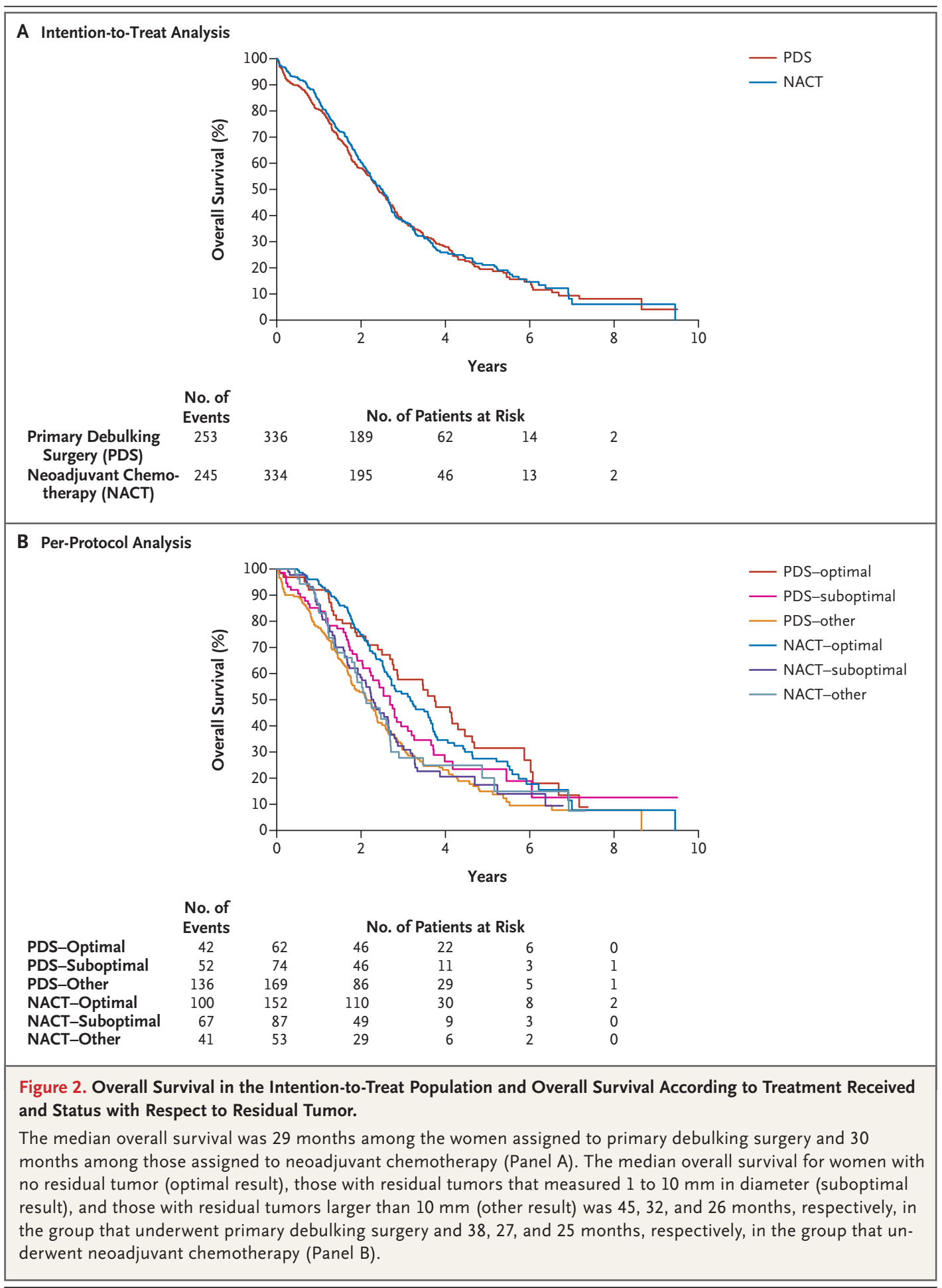

group assigned by the NCIC Clinical Trials Group (11.1\% and $40.5 \%$, respectively). Among patients slightly longer in the primary-surgery group than with metastatic tumors that were less than $5 \mathrm{~cm}$ in diameter at randomization, overall survival was in the neoadjuvant-chemotherapy group (hazard ratio, 0.64; 95\% CI, 0.45 to 0.93 ) (Fig. 10 in the Supplementary Appendix). 


\section{MULTIVARIATE ANALYSES}

Unadjusted and adjusted Cox regression multivariate analyses were performed post hoc, with overall survival as the end point, and included the following variables: largest residual tumor after primary or interval debulking surgery, largest tumor size before randomization, WHO performance status, age, FIGO stage, histologic type, method of biopsy, histologic grade, treatment group, and country (reduced to eight categories by pooling results from the smallest seven countries). The strongest independent predictors of prolonged survival, in descending order, were the absence of residual tumor after surgery $(\mathrm{P}<0.001)$; stage IIIC disease $(\mathrm{P}=0.001)$; small tumor size before randomization $(\mathrm{P}=0.001)$; endometrioid histologic type, followed in descending order by serous, mixed, undifferentiated, mucinous, and clear-cell types $(\mathrm{P}=0.005)$; and younger age $(\mathrm{P}=0.005)$. The other variables did not significantly influence overall survival.

\section{DISCUSSION}

In this randomized trial, primary debulking surgery followed by chemotherapy was compared with neoadjuvant chemotherapy followed by interval debulking surgery in women with advanced ovarian cancer. We found that survival after neoadjuvant chemotherapy followed by interval debulking surgery was similar to survival with the standard approach of primary surgery followed by chemotherapy.

This trial did not include patients with FIGO stage IIIB or earlier-stage ovarian carcinoma. Rather, all the study participants had extensive stage IIIC or IV disease. Indeed, most patients had obvious stage IIIC or IV disease; at the time of primary debulking surgery, $61.6 \%$ had metastatic lesions that were larger than $10 \mathrm{~cm}$ in diameter, and $74.5 \%$ had lesions larger than $5 \mathrm{~cm}$. This is noteworthy, since it might be the reason for the poor outcomes with respect to median progression-free and overall survival, as compared with the findings in some single-institution series ${ }^{13,15-18}$; however, the debulking rates and survival rates in our study are similar to those in other multicenter or regional studies that analyzed stage IIIC and IV ovarian carcinomas separately. ${ }^{19-25}$ Furthermore, the current study showed no trend in favor of primary debulking in countries with high rates of optimal primary debulking surgery. This finding might be due to the strong correlation between cytoreduction rates at primary debulking surgery and at interval debulking surgery within each country.

In selecting patients for neoadjuvant chemotherapy, it is important to rule out other primary tumors, especially those of gastrointestinal origin. In this study, a CA-125:CEA ratio higher than 25 was used as an eligibility criterion, since this ratio has been shown to be a good tool for ruling out primary gastrointestinal tumors that have metastasized to the peritoneum or ovaries or to both sites. ${ }^{9}$ The value of this ratio was confirmed in the current study, since only two patients proved to have a gastrointestinal cancer at the time of primary or interval debulking surgery.

Complete resection of all macroscopic disease at primary debulking surgery has been shown to be the single most important independent prognostic factor in advanced ovarian carcinoma. ${ }^{13,15-18,25,26}$ In the current study, the importance of this prognostic factor was confirmed by the results of the multivariate analyses and the survival analyses according to the extent of residual tumor after both primary and interval debulking surgery (Fig. 2B). Given our findings and the results of other studies, a potential approach for debulking surgery could be the elimination of all macroscopic residual disease, rather than the elimination of lesions larger than $1 \mathrm{~cm}$ in diameter. A potential drawback of neoadjuvant chemotherapy followed by debulking surgery is that the occurrence of fibrosis after chemotherapy may make complete resection of macroscopic disease more difficult.

In conclusion, among patients with advanced (stage IIIC or IV) ovarian, fallopian-tube, or peritoneal ovarian carcinoma, survival after neoadjuvant chemotherapy followed by interval debulking surgery is similar to survival after primary debulking surgery followed by chemotherapy. This result is consistent with the conclusions of a recent meta-analysis of 21 nonrandomized trials. ${ }^{27}$ The standard of care for women with stage IIIB or earlier-stage epithelial ovarian cancer - a group with a better prognosis than the current study population - remains primary cytoreductive surgery. Only those patients with proven stage IIIC or IV disease should be considered for neoadjuvant chemotherapy.

In the current study, none of the subgroup 
analyses showed a significant difference in survival between the two treatment groups. When deciding whether a patient is a candidate for primary debulking surgery, with an acceptable level of morbidity, the clinician may consider taking into account information from the surgical consultation and could assess important predictive factors with respect to residual macroscopic disease after debulking surgery (e.g., presence or absence of coexisting illnesses, age, disease burden, location of metastatic sites, WHO performance status, and tumor stage). Laparoscopy, in addition to axial CT, positron-emission tomography, or both, ${ }^{28}$ may provide information about the disease burden. ${ }^{29-32}$ Neoadjuvant chemother- apy is not inferior to primary cytoreductive surgery for patients with stage IIIC or IV ovarian carcinoma. No significant advantages of neoadjuvant therapy or primary debulking surgery were observed with respect to survival, adverse effects, quality of life, or postoperative morbidity or mortality.

The views expressed in this article are those of the authors and do not necessarily reflect the official views of the National Cancer Institute.

Supported by grants (2U10 CA11488-28 through $2 \mathrm{U} 10$ CA011488-36) from the National Cancer Institute and by a donation from Vlaamse Liga Tegen Kanker (the Flemish League against Cancer) to the EORTC Charitable Trust.

Disclosure forms provided by the authors are available with the full text of this article at NEJM.org.

\section{APPENDIX}

In addition to the authors, the following EORTC-GCG and NCIC Clinical Trials Group collaborators participated in the study: R. Angioli (Università Campus BioMedico di Roma, Rome), J. Bentley (Nova Scotia Cancer Centre, Halifax, Canada), P. Berteloot (University Hospital Leuven, Leuven, Belgium), P. Bessette (Centre Hospitalier Universitaire de Sherbrooke, Sherbrooke, QC, Canada), K. Boman (Umeå University, Umeå, Sweden), M. Buist (Academic Medical Center, Amsterdam), K. Chan (Hôpital Charles Lemoyne, Longueuil, QC, Canada), S. Chan (Nottingham City Hospital, Nottingham, United Kingdom [UK]), P. Coronado Martín (Hospital Universitario San Carlos, Madrid), R. Counsell (Cheltenham General Hospital, Cheltenham, UK), D.J. Cruickshank (James Cook University Hospital, Middlesbrough, UK), J. Davis (Gartnavel General Hospital and Beatson Oncology Center, Gynaecological Oncology, Glasgow, UK), J. De Greve (Universitair Ziekenhuis Brussel, Brussels), C.F. De Oliveira (Hospitais sa Universidade de Coimbra, Coimbra, Portugal), B. De Valk (Onze Lieve Vrouw Gasthuis, Amsterdam), C. Dittrich (Kaiser Franz Josef Spital, Vienna), L. Elit (Hamilton Health Sciences, Juravinski Cancer Centre, Hamilton, ON, Canada), G. Favalli (Ospedale Sta Maria Delle Croci, Ravenna, Italy), A. Floquet (Insititut Bergonie, Bordeaux, France), P. Gauthier (Hôpital Notre-Dame du CHUM, Montreal), E. Gerdin (Akademiska Sjukhuser, Uppsala, Sweden), P. Ghatage (Tom Baker Cancer Centre, Calgary, AB, Canada), E. Gilby (Royal United Hospital, Bath, UK), N. Gleeson (Coombe Women's Hospital, Dublin), W. Gotlieb (McGill University, Montreal), J.A. Green (Clatterbridge Center for Oncology National Health Service Trust, Liverpool, UK), R. Grimshaw (Nova Scotia Cancer Centre, Halifax, Canada), M. Heywood (CancerCare Manitoba, Winnipeg, Canada), V. Hirsch (McGill University, Montreal), K. Hoekman (Vrije Universitei Medical Center, Amsterdam), A. Honkoop (Sophia Ziekenhuis, Zwolle, the Netherlands), P. Hoskins (British Columbia Cancer Agency [BCCA]-Vancouver Cancer Centre, Vancouver, BC, Canada), P. Kannisto (Lund University Hospital, Lund, Sweden), J. Kaern (Norwegian Radium Hospital, Oslo), D. Katsaros (Clinica Universita, Turin, Italy), K. Kieser (Nova Scotia Cancer Centre, Halifax, Canada), T.V. Kristeller (I.P.O. Francisco Gentil Centro de Lisboa, Lisbon, Portugal), E. Leblanc (Centre Oscar Lambret, Lille, France), J. Ledermann (University College Hospital, London), K. Leunen (University Hospital Leuven, Leuven, Belgium), R. Lotocki (CancerCare Manitoba, Winnipeg, Canada), T. Maggino (Mirano General Hospital-Veneto, Mirano, Italy), C. Marth (Innsbruck Universitaetsklinik, Innsbruck, Austria), L. Martin (BCCA-Fraser Valley Cancer Centre, Surrey, BC, Canada), L. Massuger (Radboud University Nijmegen Medical Center, Nijmegen, the Netherlands), D. Miller (BCCA-Vancouver Cancer Centre, Vancouver, BC, Canada), B. Mosgaard (Herlev Hospital-University of Copenhagen, Copenhagen) F. Mota (Hospitais da Universidaded de Coimbra, Coimbra, Portugal), P. Neven (University Hospital Leuven, Leuven, Belgium), M. Nooij (Leiden University Medical Center, Leiden, the Netherlands), R. Nordal (Haukeland Hospital-University of Bergen, Bergen, Norway), A. Nordin (Queen Elizabeth, the Queen Mother Hospital, Margate [Kent], UK), P.B. Ottevanger (Radboud University Nijmegen Medical Centre, Nijmegen, the Netherlands), A. Papadopoulos (Mid Kent Oncology Centre, Maidstone [Kent], UK), E. Petru (Medical University of Graz, Graz, Austria), M. Plante (Centre Hospitalier de l'Université de Québec-Pavillon Hotel-Dieu de Québec, Quebec City, QC, Canada), C. Popadiuk (Dr. H. Bliss Murphy Cancer Centre, St. John's, NF, Canada), D. Provencher (Hôpital Notre-Dame du Centre Hospitalier de l'Université de Montréal, Montreal), C. Redman (North Staffordshire Hospital, Staffordshire, UK), K.J. Roozendaal (Onze Lieve Vrouw Gasthuis, Amsterdam), G. Rustin (Mount Vernon Hospital, Northwood, Middlesex, UK), A.H. Sadozye (Gartnavel General Hospital, Glasgow, UK), R. Sandvei (Haukeland Hospital, University of Bergen, Bergen, Norway), J.M. Seoane (Universitario 12 de Octubre, Madrid), M.I. Sereni (Campus BioMedico, University of Rome, Rome), B. Sert (Norwegian Radium Hospital, Oslo), N. Siddiqui (Royal Infirmary, University of Glasgow, Glasgow, UK), P. Speiser (Allgemeines Krankenhaus der Stadt Wien, Vienna), B. Tholander (Karolinksa University Hospital, Stockholm), G. Tognon (Universita di Brescia, Brescia, Italy), B. Trimbos (Leiden University Medical Center, Leiden, the Netherlands), M. Trudeau (McGill University, Montreal), M. Van Baal (Vrije Universiteit Medisch Centrum, Amsterdam), H.C. Van Doorn (Erasmus MC University Medical Center Rotterdam, Rotterdam, the Netherlands), J. Van Der Velden (Academisch Medisch Centrum, Amsterdam), K. Van Eygen (AZ Groeninghe, Campus Maria's Voorzienigheid, Kortrijk, Belgium), J.B. Vermorken (Universitair Ziekenhuis Antwerpen, Antwerp, Belgium), J.A. Vidart Aragon (Hospital Universitario San Carlos, Madrid), C.W.M. Wensveen (Erasmus MC University Medical Center Rotterdam, Rotterdam, the Netherlands), P. Zola (Ospedale Mauriziano Umberto I, Turin, Italy). EORTC Headquarters, Brussels - A. Anastosopoulou, U. Bethe, K. Dehaes, A. Demeester, G. Demonty, E. De Heusch, M. De Rouck, L. Giurgea, G. Hoctin-Boes I. Teodorovic, K. Ven, I. Van Luijk. NCIC Clinical Trials Group Headquarters, Queen's University, Kingston, ON, Canada - M. Bacon, E. Eisenhauer. 
REFERENCES

1. Meigs JV. Tumors of the pelvic organs. New York: Macmillan, 1934.

2. Aure JC, Hoeg K, Kolstad P. Clinical and histologic studies of ovarian carcinoma: long-term follow-up of 990 cases. Obstet Gynecol 1971;37:1-9.

3. Griffiths CT, Fuller AF. Intensive surgical and chemotherapeutic management of advanced ovarian cancer. Surg Clin North Am 1978;58:131-42.

4. du Bois A, Quinn M, Thigpen T, et al. 2004 Consensus statements on the management of ovarian cancer: final document of the 3rd International Gynecologic Cancer Intergroup Ovarian Cancer Consensus Conference (GCIG OCCC 2004). Ann Oncol 2005;16:Suppl 8:viii7-viii12.

5. van der Burg MEL, van Lent M, Buyse $M$, et al. The effect of debulking surgery after induction chemotherapy on the prognosis in advanced epithelial ovarian cancer. N Engl J Med 1995;332:629-34.

6. Rose PG, Nerenstone S, Brady MF, et al. Secondary surgical cytoreduction for advanced ovarian carcinoma. N Engl J Med 2004;351:2489-97.

7. Vergote I, van Gorp T, Amant F, Leunen K, Neven P, Berteloot P. Timing of debulking surgery in advanced ovarian cancer Int J Gynecol Cancer 2008;18:Suppl 1:11-9. 8. Bristow RE, Chi DS. Platinum-based neoadjuvant chemotherapy and interval surgical cytoreduction for advanced ovarian cancer: a meta-analysis. Gynecol Oncol 2006;103:1070-6.

9. Yedema CA, Kenemans P, Wobbes T, et al. Use of serum tumor markers in the differential diagnosis between ovarian and colorectal adenocarcinomas. Tumour Biol 1992;13:18-26.

10. WHO handbook for reporting results of cancer treatments. Geneva: World Health Organization, 1979. (WHO offset publication no. 48 .)

11. Miller AB, Hoogstraten B, Staquet M, Winkler A. Reporting results of cancer treatment. Cancer 1981;47:207-14.

12. Vergote I, Rustin GJ, Eisenhauer EA, et al. Re: new guidelines to evaluate the response to treatment in solid tumors [ovarian cancer]. J Natl Cancer Inst 2000; 92:1534-5.

13. Vergote I, De Wever I, Tjalma W, Van Gramberen M, Decloedt J, van Dam P. Neoadjuvant chemotherapy or primary debulking surgery in advanced ovarian carcinoma: a retrospective analysis of 285 patients. Gynecol Oncol 1998;71:431-6.
14. Strategy for model selection. In: Collett $\mathrm{D}$. Modelling survival data in medical research. London: Chapman \& Hall, 1994: 78-86.

15. Zivanovic O, Eisenhauer EL, Zhou Q, et al. The impact of bulky upper abdominal disease cephalad to the greater omentum on surgical outcome for stage IIIC epithelial ovarian, fallopian tube, and primary peritoneal cancer. Gynecol Oncol 2008;108:287-92.

16. Aletti GD, Dowdy SC, Podratz KC, Cliby WA. Surgical treatment of diaphragm disease correlates with improved survival in optimally debulked advanced stage ovarian cancer. Gynecol Oncol 2006; 100:283-7.

17. Bristow RE, Tomacruz RS, Armstrong DK, Trimble EL, Montz FJ. Survival effect of maximal cytoreductive surgery for advanced ovarian carcinoma during the platinum era: a meta-analysis. J Clin Oncol 2002;20:1248-59.

18. Chi DS, Eisenhauer EL, Land J, et al. What is the optimal goal of primary cytoreductive surgery for bulky stage IIIC epithelial ovarian carcinoma (EOC)? Gynecol Oncol 2006;103:559-64.

19. O'Malley CD, Cress RD, Campleman SL, Leiserowitz GS. Survival of Californian women with epithelial ovarian cancer, 1994-1996: a population-based study. Gynecol Oncol 2003;91:608-15.

20. Schrag D, Earle C, Xu F, et al. Associations between hospital and surgeon procedure volumes and patient outcomes after ovarian cancer resection. J Natl Cancer Inst 2006;98:163-71.

21. Heintz APM, Odicino F, Maisonneuve $\mathrm{P}$, et al. Carcinoma of the ovary: FIGO 6th Annual Report on the Results of Treatment in Gynecological Cancer. Int J Gynaecol Obstet 2006;95:Suppl 1:S161-S191. 22. Crawford SC, Vasey PA, Paul J, Hay A, Davis JA, Kaye SB. Does aggressive surgery only benefit patients with less advanced cancer? Results from an international comparison within the SCOTROC-1 Trial. J Clin Oncol 2005;23:8802-11. [Erratum, J Clin Oncol 2006;24:1224.]

23. Vernooij F, Heintz AP, Witteveen PO, van der Heiden-van der Loo M, Coebergh JW, van der Graaf Y. Specialized care and survival of ovarian cancer patients in the Netherlands: nationwide cohort study. J Natl Cancer Inst 2008;100:399-406. 24. Marth C, Hiebl S, Oberaigner W, Winter R, Leodolter S, Sevelda P. Influence of department volume on survival for ovarian cancer: results from a prospective quality assurance program of the Austrian Association for Gynecologic Oncology. Int J Gynecol Cancer 2009;19:94-102.

25. du Bois A, Reuss A, Pujade-Lauraine E, Harter P, Ray-Coquard I, Pfisterer J. Role of surgical outcome as prognostic factor in advanced epithelial ovarian cancer: a combined exploratory analysis of 3 prospectively randomized phase 3 multicenter trials: by the Arbeitsgemeinschaft Gynaekologische Onkologie Studiengruppe Ovarialkarzinom (AGO-OVAR) and the Groupe d'Investigateurs Nationaux Pour les Etudes des Cancers de l'Ovaire (GINECO). Cancer 2009;115:1234-44.

26. Eisenkop SM, Friedman RL, Wang HJ. Complete cytoreductive surgery is feasible and maximizes survival in patients with advanced ovarian cancer: a prospective study. Gynecol Oncol 1998;69:103-8.

27. Kang S, Nam BH. Does neoadjuvant chemotherapy increase optimal cytoreduction rate in advanced ovarian cancer? Meta-analysis of 21 studies. Ann Surg Oncol 2009;16:2315-20.

28. Risum S, H申gdall C, Loft A, et al. Prediction of suboptimal primary cytoreduction in primary ovarian cancer with combined positron emission tomography/ computed tomography - a prospective study. Gynecol Oncol 2008;108:265-70.

29. Vergote I, Marquette S, Amant F, Berteloot P, Neven P. Port-site metastases after open laparoscopy: a study in 173 patients with advanced ovarian carcinoma. Int J Gynecol Cancer 2005;15:776-9.

30. Fagotti A, Fanfani F, Ludovisi M, et al. Role of laparoscopy to assess the chance of optimal cytoreductive surgery in advanced ovarian cancer: a pilot study. Gynecol Oncol 2005;96:729-35.

31. Angioli R, Palaia I, Zullo MA, et al. Diagnostic open laparoscopy in the management of advanced ovarian cancer. Gynecol Oncol 2006;100:455-61.

32. Brun JL, Rouzier R, Uzan S, Daraï E. External validation of a laparoscopic-based score to evaluate resectability of advanced ovarian cancers: clues for a simplified score. Gynecol Oncol 2008;110:354-9. Copyright (@ 2010 Massachusetts Medical Society.

COLLECTIONS OF ARTICLES ON THE JOURNAL'S WEB SITE

The Journal's Web site (NEJM.org) sorts published articles into more than 50 distinct clinical collections, which can be used as convenient entry points to clinical content. In each collection, articles are cited in reverse chronologic order, with the most recent first. 